\title{
«Wenn einer Patient sein muss, dann kann er was erleben ...»
}

Monika M. Käppeli

\section{Der Arzt stellte meine Antworten in Frage mit Bemer- kungen wie: «Das kann doch eigentlich gar nicht sein!»}

Der behandelnde Gastroenterologe hat mir mit Begeisterung sehr viel Positives über diese Untersuchung und den sie durchführenden Experten erzählt, was mich bewogen hat, mich - trotz aller erahnbarer Unannehmlichkeiten - anmelden zu lassen.

Ich erhalte also ein entsprechendes Aufgebot mit schriftlichen Anweisungen, was vorbereitend zu tun sei. Bereits diese verunsichern mich und veranlassen mich zu einem Telefonanruf, enthalten doch Broschüre und Begleitbrief unterschiedliche Angaben da$\mathrm{zu}$, wie lange vorher man die Säureblocker abzusetzen habe und wie lange vor dem Untersuch Essen und Trinken nicht mehr erlaubt seien. Ich habe die Auskünfte also befolgt und stehe am vereinbarten Termin an der Anmeldung, nüchtern, eine längere Reisezeit hinter mir, erwartungsvoll.

Zuerst warte ich einmal eine gute halbe Stunde. Dauernd laufen Menschen in medizinischer Berufskleidung an mir vorbei, ohne dass mir jemand zu verstehen gäbe, dass ich zur Untersuchung vorgesehen bin. Unsicherheit beschleicht mich. Bin ich hier richtig?

Schliesslich werde ich von einer Fachfrau, deren Qualifikation ich nicht kenne, in ein Behandlungszimmer gebeten. Über sie kann ich mich uneingeschränkt positiv äussern. Sie begegnet mir freundlich und einfühlsam.

Dann kommt der Arzt dazu. Er begrüsst mich knapp und wendet sich dann den Zuweisungsberichten zu, die er offensichtlich noch nicht gelesen hat. Er ist denn auch nicht informiert, was alles zu tun sei, und meint anscheinend, es stünden noch andere Untersuchungen an; er bemerkt, die könnten aber nicht alle heute durchgeführt werden ...

Irgendwann geht er dazu über, mir anamnestische Fragen zu stellen. Anstatt mir zuzuhören, stellt der Arzt meine Antworten in Frage oder in Abrede mit Bemerkungen wie: «Das kann doch eigentlich gar nicht sein.» In seinen Äusserungen ist ein verärgerter, verhalten-aggressiver Unterton nicht zu überhören. Meine Antworten scheinen nicht dem zu entsprechen, was er erwartet. Was habe ich falsch gemacht?

Schliesslich geht der Arzt - er trägt den Rang eines PD Dr. med. - zur Untersuchung über. Ich bekomme keinerlei Informationen darüber, was er nun an mir zu machen gedenkt, und ebenso wenig darüber, welche Mitarbeit dazu von mir gefordert ist. Die relativ dicke Manometrie-Sonde wird gesteckt. Erst bei der dritten Rüge darüber, dass ich geschluckt hätte, «sogar zweimal», geht mir ein Licht auf: Ich darf offenbar nicht schlucken oder jedenfalls nur dann, wenn ich dazu aufgefordert werde. Es braucht eine weitere Massregelung, bis ich herausfinde, dass ich beim Befehl, zu schlucken, die ganze Wassermenge in einem einzigen Mal hinunterkriegen muss.

Ich komme mir schon völlig hilflos und ausgeliefert vor. Tränen laufen mir aus den Augen, Rotz aus der Nase, der Hals (und die Seele) schmerzen. So kann ich in dieser Situation nichts darüber sagen, was ich brauchen würde.

Die Arzthelferin reicht mir ein Tüchlein. Wie sehr würden ein empathischer Zuspruch und klare Informationen seitens des Arztes helfen!

Ich bekomme den Eindruck, dass er sich in keinem Augenblick bewusst ist, dass das, was er macht (und ja auch machen muss), für mich total unangenehm und schmerzhaft ist, dass mir mein Zustand peinlich ist und dass ich mich wehrlos, hilflos und ohnmächtig fühle. Mir geht durch den Kopf, dass jeder handelnde Arzt diese Prozeduren mal in der Patientenrolle erlebt haben müsste.

Gott sei Dank wird die Manometrie-Sonde wieder gezogen. Ich nehme das mit Erleichterung zur Kennt- 


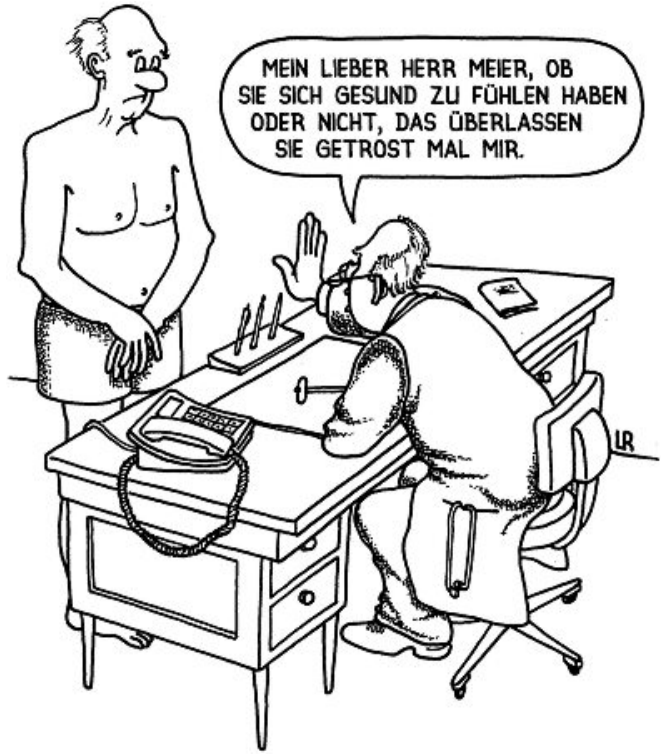

nis, denn ich habe mich schon die ganze Zeit gefragt, wie ich es machen sollte, um diesen dicken Fremdkörper über die nächsten 24 Stunden aushalten zu können. Nun wird über meinen Kopf hinweg über die Sondenlänge verhandelt: Man hoffe, dass sie lang genug gewesen sei. Ich hätte einen doch sehr langen Ösophagus. Und was die pH-Messsonde betreffe: Da sei leider keine passende im Haus. Sie sei wohl bestellt worden, aber nicht eingetroffen. Man würde es mit einer Sonde probieren, die sonst nicht verwendet werde, und hoffe, dass es klappe. Andernfalls müsse man halt alles wiederholen ...

Das sagt sich so leicht. Ich wünschte mir, die Spiegelneuronen dieses Arztes wären wenigstens zur Hälfte so aktiv wie die meinen ... In mir läuft ein Film der bereits erlittenen Strapazen und eingegangenen Konzessionen ab. Ich habe die Säureblocker seit zehn Tagen abgesetzt, nicht ohne spürbare Folgen. Ich habe seit gestern nichts mehr gegessen und getrunken und es geht gegen Mittag. Ich habe allen Patienten meiner Praxis für diese zwei Untersuchungstage abgesagt. Ich habe eine Reise von eineinhalb Stunden hinter (und vor) mir, und meine Begleitperson hat sich für ihre Taxidienste an diesen zwei Tagen ebenfalls von beruflichen Verpflichtungen freigemacht. Ob denn dieser Arzt eine Vorstellung davon hat?

Als nächstes wird mir, auch wieder ohne Erklärung, die zum Glück deutlich dünnere Ersatz-pH-Messsonde eingelegt. Das Messgerät wird angehängt. Es zeigt Knöpfe, die registrieren können, ob man in aufrechter oder liegender Position ist, ob man gerade isst oder nicht, ob man Druckgefühle, ein Aufstossen von Luft oder Flüssigkeit registriert und einen Extraknopf, der mit «diary» beschriftet ist. Ich erhalte Erläuterungen über die technische Funktion der verschiedenen Knöpfe, nicht aber darüber, was ich denn nun konkret damit anfangen soll, und ebenso wenig darüber, dass ich wohl einen Begleitzettel schreiben soll für die Option «diary-Tagebucheinträge», die für andere, bemer- kenswerte Mitteilungen vorgesehen ist. Also versuche ich, eins plus eins selber zusammenzuzählen und herauszufinden, was ich in den kommenden 24 Stunden mit diesem Gerät alles registrieren soll. Dann bin ich entlassen. Ich trete vors Haus und nehme wahr, dass mich viele neugierige Blicke unverhohlen anschauen.

Am kommenden Tag bin ich pünktlich um $11 \mathrm{Uhr}$ wieder da. Am Eingang der Warteecke kreuze ich die mir bekannte Arzthelferin. Sie begrüsst mich freundlich und sagt, sie komme gleich, um mir die Sonde zu entfernen. Der Herr Doktor läuft an mir vorbei, ohne Gruss, verschwindet in einem Büro, kommt wenig später zurück. Er geht auf mich zu, grüsst und meint: «Ist es gut gegangen?» Ich verstehe das als Frage und setze zu einer Antwort an. Da merke ich aber schon, dass sein Ausspruch nicht als Frage, sondern als Postulat $\mathrm{zu}$ verstehen ist. Und schon wieder stellt er meine Schilderung in Abrede. Er hat offenbar erwartet, dass ich die Sonde leicht ertragen und gut geschlafen habe und dass es gut möglich gewesen sei, die Symptome des Grundleidens klar wahrzunehmen und zu registrieren. Ich versuche zu erklären, dass ich die schmerzhaft-drückende Irritation der Sonde als so dominant erlebe, dass dadurch schlicht manch andere Wahrnehmung überlagert wird. Gleich bekomme ich zu hören, dass das sonst eigentlich nicht üblich sei.

Die Arzthelferin kommt dazu und erhält vom Arzt in ungehaltenem Ton den Auftrag, mir die Sonde zu ziehen. Das will sie ja aber ohnehin schon tun. Mit Sorgfalt befreit mich die Assistentin von der Sonde und macht dabei eine Bemerkung, die durchblicken lässt, dass sie es diesem Arzt kaum recht machen kann.

Diese eine Bemerkung der Mitarbeiterin des Arztes hat mich in meinem Erleben bestätigt. (Ich war nämlich dabei, mich zu fragen, ob ich denn wirklich so übersensibel sei und Sachen wahrzunehmen meine, die nicht der Realität entsprechen.)

Ich entscheide mich daraufhin, der Fachbereichsleitung meine Erfahrungen mitzuteilen.

Auf die Auswertung der Aufzeichnungen warte ich nun gespannt. Ich habe die nicht bescheidene Hoffnung, dass ich eine brauchbare therapeutische Option aufgezeigt bekomme, die mir einen Teil der verlorengegangenen Lebensqualität zurückgibt.

Ich danke Ihnen, dass Sie sich die Zeit genommen haben, meine Aufzeichnungen zu lesen. Was mich dieses Erlebnis als Ärztin gelehrt hat? Zwei Sachen, glaube ich:

Erstens: Empfehlungen sind eine heikle Sache. Anscheinend kann ich nicht garantieren, dass meine Patientinnen und Patienten von den Personen behandelt werden, die ich eigentlich empfehle und von deren Arbeitsweise und Kompetenz ich überzeugt bin.

Zweitens: Als Ärztin kann ich mir nie genügend bewusst sein, wie sensibel und verletzlich man in der Rolle als Patientin ist. Dies ist sogar so, wenn man dem gleichen Berufsstand angehört wie die Behandelnden ... 